\title{
Terminal ileum ileoscopy and histology in patients undergoing high-definition colonoscopy with virtual chromoendoscopy for chronic nonbloody diarrhea: A prospective, multicenter study
}

United European Gastroenterology Journa 2019, Vol. 7(7) 974-981 (C) Author(s) 2019 Article reuse guidelines: sagepub.com/journals-permissions DOI: $10.1177 / 2050640619847417$ journals.sagepub.com/home/ueg (S)SAE

\author{
Edoardo Borsotti ${ }^{1}$ (D, Brigida Barberio ${ }^{2}$, Renata D'Incà ${ }^{2}$, Gianluca Bonitta ${ }^{1}$, \\ Flaminia Cavallaro ${ }^{1}$, Luca Pastorelli ${ }^{1,3}$, Emanuele Rondonotti ${ }^{4}$, \\ Leonardo Samperi ${ }^{5}$, Helmut Neumann ${ }^{6}$, Chiara Vigano ${ }^{7}$, Maurizio Vecchi, ${ }^{1,8,9}$ \\ and Gian Eugenio Tontini, ${ }^{1,8}$
}

\begin{abstract}
Background and aims: lleo-colonoscopy is the procedure of choice for chronic nonbloody diarrhea (CNBD) of unknown origin. Histological evaluation at different colonic sites is mandatory to assess the presence of microscopic colitis. However, the value of routine ileal biopsy on normal-appearing mucosa as assessed by means of standard-resolution white-light ileoscopy is controversial given its reported low diagnostic yield. Hence, we have assessed for the first time the accuracy of retrograde ileoscopy using high-definition and dyeless chromoendoscopy (HD + DLC), thereby calculating the impact and cost of routine ileal biopsy in CNBD. Methods: Patients with CNBD of unknown origin were prospectively enrolled for ileo-colonoscopy with $\mathrm{HD}+\mathrm{DLC}$ at five referral centers. Multiple biopsies were systematically performed on each colorectal segment and in the terminal ileum for histopathological analysis.

Results: Between 2014 and 2017, 546 consecutive patients were recruited. Retrograde ileoscopy success rate was $97.6 \%$. A total of 492 patients (mean age: $53 \pm 18$ years) fulfilled all the inclusion criteria: Following endoscopic and histopathological workup, $7 \%$ had lymphoid nodular hyperplasia and $3 \%$ had isolated ileitis. Compared to the histopathology as the gold standard, retrograde ileoscopy with $\mathrm{HD}+\mathrm{DLC}$ showed $93 \%$ sensitivity, $98 \%$ specificity and $99.8 \%$ negative predictive value. In patients with normal ileo-colonoscopy, ileum histology had no diagnostic gain and resulted in a cost of US \$26.5 per patient.

Conclusions: Retrograde ileoscopy with HD + DLC predicts the presence of ileitis in CNBD with excellent performance. The histopathological evaluation of the terminal ileum is the gold standard for the diagnostic assessment of visible lesions but has no added diagnostic value in CNBD patients with negative ileo-colonoscopy inspection using modern endoscopic imaging techniques.
\end{abstract}

\section{Keywords}

Advanced endoscopic imaging, chronic nonbloody diarrhea, colonoscopy, Crohn disease, high-definition, histopathology, ileal biopsy, ileoscopy, virtual chromoendoscopy

Received: 9 January 2019; accepted: 22 March 2019

\footnotetext{
${ }^{1}$ Gastroenterology and Digestive Endoscopy Unit, IRCCS Policlinico San Donato, San Donato Milanese, Milan, Italy

${ }^{2}$ Department of Surgery, Oncology and Gastroenterology, University of Padua, Padua, Italy

${ }^{3}$ Department of Biomedical Sciences for Health, University of Milan, Milan, Italy

${ }^{4}$ Gastroenterology Unit, Ospedale Valduce, Como, Italy

${ }^{5}$ Gastroenterology and Digestive Endoscopic Unit, Ospedale Morgagni Pierantoni, Forli, Italy

${ }^{6}$ Department of Interdisciplinary Endoscopy, 1st Medical Clinic and Polyclinic, University Hospital Mainz, Mainz, Germany
}

${ }^{7}$ Gastroenterology Division, San Gerardo Hospital, ASST Monza, Monza, Italy

${ }^{8}$ Gastroenterology and Endoscopy Unit, IRCCS Ca' Granda Ospedale Maggiore Policlinico Foundation, Milan, Italy

${ }^{9}$ Department of Pathophysiology and Transplantation, University of Milan, Milan, Italy

Corresponding author:

Gian Eugenio Tontini, Fondazione IRCCS Ca' Granda Ospedale Maggiore Policlinico, Gastroenterology and Endoscopy Unit, Via della Commenda 12, 20122 Milan, Italy.

Email: gianeugeniotontini@gmail.com 


\section{Key summary}

1. In the work-up for chronic nonbloody diarrhea (CNBD) of unknown origin, ileo-colonoscopy with histological evaluation at different colonic sites is mandatory.

- Histopathological evaluation of the terminal ileum is the gold standard for diagnostic assessment of visible lesions.

- By contrast, the value of routine ileal biopsy on normal-appearing mucosa is controversial.

2. The histopathological evaluation of the terminal ileum has no added diagnostic value in CNBD patients with negative ileo-colonoscopy inspection using modern endoscopic imaging techniques.

- Retrograde ileoscopy with high-definition and dyeless chromoendoscopy predicts the presence of ileitis in CNBD with excellent performance.

\section{Introduction}

Retrograde ileoscopy with biopsy is widely recognized as a key element for the diagnostic evaluation of chronic diarrhea, especially in patients suspected of having inflammatory illness. ${ }^{1-3}$ The differential diagnosis for abnormal endoscopic and histological findings in the terminal ileum includes Crohn disease, druginduced enteropathy, carcinoid, tuberculosis, yersiniosis, lymphoma and adenocarcinoma. ${ }^{1,3-6}$ Histology may be of greatest value when an abnormal terminal ileum is identified endoscopically or via an imaging study. ${ }^{3,6,7}$ However, the value of ileal biopsy on normal-appearing mucosa is controversial in the routine assessment of patients suffering from chronic diarrhea, with significant findings ranging from $0 \%$ to $4.2 \%$ of retrograde ileoscopy performed with standard-resolution white-light endoscopy. ${ }^{1,5,7-10}$

More recently, high-definition (HD) endoscopy systems, integrated with optical or digital virtual chromoendoscopy, have become widely available in daily clinical practice. ${ }^{10-12}$ These modern endoscopic imaging techniques improve the detection of subtle lesions by providing high-quality mucosal-surface and vascularpattern imaging, thereby enabling more precise and on-demand targeted biopsy. ${ }^{13}$

Accordingly, the aim of this prospective, multicenter trial was first to assess the diagnostic accuracy of retrograde ileoscopy with HD and dyeless chromoendoscopy in patients with chronic nonbloody diarrhea (CNBD) of unknown origin using histopathology as the gold standard, and second to determine the diagnostic yield and cost of routine biopsies of the terminal ileum for histopathological assessment beyond normalappearing mucosa in CNBD.

\section{Methods}

\section{Patients}

A prospective cohort study was performed across five tertiary referral centers in northern Italy (Como, Forli, Monza, Padua and San Donato Milanese). Adults referred for colonoscopy were consecutively enrolled according to the following inclusion criteria:

- CNBD of unknown origin, defined by more than three loose or liquid nonbloody stools per day, for a minimum of four consecutive weeks. ${ }^{1}$

- Absence of active disorders that correspond to one of the following: celiac disease, lactose/fructose intolerance, known inflammatory bowel disease (IBD).

- No severe uncontrolled coagulopathy, pregnancy or breastfeeding.

Patients with one of the following criteria were excluded from the study analysis:

- Boston bowel preparation scale $\geq 2$ in any segment.

- History of large-bowel or small-bowel surgery.

- Incomplete ileo-colonoscopy with biopsy or incomplete image documentation.

\section{Endoscopic procedure}

All colonoscopies were performed under conscious or deep sedation by experienced endoscopists, using HD and virtual chromoendoscopy systems including either optical (Narrow Band Imaging, NBI, Olympus, Tokyo, Japan) or digital technologies (i-SCAN, Pentax, Tokyo, Japan; Storz Professional Image Enhancement Systems, SPIES, Karl Storz, Tuttlingen, Germany) according to local availability. Endoscopic incidents and adverse events were prospectively recorded using the American Society for Gastrointestinal Endoscopy (ASGE) lexicon. ${ }^{14}$

As per protocol, a clear, detailed description of every segment was performed, including at least two images demonstrating a stable ileoscopy visualization for at least 30 seconds by means both of HD white-light endoscopy and virtual chromoendoscopy. Afterward, no fewer than two large biopsies (i.e. 9-mm diameter of opened jaws, e.g. Radial Jaw 4 Biopsy Forceps, Boston Scientific, Natick, MA, USA, or similar) of a 
grossly normal mucosa were randomly performed in the terminal ileum (usually $5-15 \mathrm{~cm}$ proximal to the ileocecal valve), right colon, transverse colon, left colon and rectum. Additional targeted samples were systematically collected from any visible endoscopic lesion. Biopsy specimens were immediately fixed in buffered formalin and stored in separate vials to map any endoscopic and microscopic finding in different ileocolonic segments.

\section{Histopathological analysis}

Ileocolorectal biopsy specimens underwent identical sectioning and hematoxylin and eosin staining followed by specific additional staining when needed (e.g. immunohistochemistry, trichrome). Histopathological analysis was performed by two dedicated subspecialty-trained gastrointestinal pathologists at each tertiary referral center involved in the present study. The histopathological diagnostic definition of microscopic colitis, ${ }^{15}$ IBD,${ }^{16}$ eosinophilic ${ }^{17}$ or drug-induced enterocolitis ${ }^{18}$ followed the established standard of care outlined in the literature. The terms nonspecific colitis or ileitis were adopted for moderate-to-severe microscopic inflammatory tissue changes without a definitive diagnosis (i.e. increased lamina propria cellularity without cryptitis, apoptotic bodies or features of "incomplete microscopic colitis"). ${ }^{3,15,16,18}$

\section{Ethics}

All patient records (inclusion/exclusion criteria, endoscopic and histopathological findings) were anonymized and prospectively collected in a structured database. Each center received formal approval from the local ethics committee before study initiation. The patients gave their informed written informed consent prior to ileocolonoscopy for the present trial. This study was carried out in accordance with the Declaration of Helsinki adopted in 1964 incorporating all later amendments.

\section{Statistical analysis}

The descriptive statistics were presented as means $\pm \mathrm{SD}$ for continuous variables and percentages for categorical variables. We estimated diagnostic variables such as sensibility, specificity, likelihood ratios and relative confidence intervals (which were calculated using the 95\% confidence level (CI)). Statistical analysis was carried out by R software.

\section{Cost analysis}

For the estimation of the terminal ileum histopathological assessment cost, we searched the database of one Italian referral center (Foundation IRCCS Ca' Granda Ospedale Maggiore Policlinico, University of Milan) and one United States (US) referral center (Cleveland Digestive Diseases Research Core Center, Cleveland, $\mathrm{OH}$, Case Western Reserve University) asking about the following costs: (a) disposable container preloaded with buffered formalin; (b) standard slide preparation (specimen embedding, sectioning, hematoxylin and eosin staining); (c) standard slide pathology review; and (d) specimen sectioning for two additional staining and additional pathological review for special situations (e.g. difficult diagnostic definition of terminal ileum entities in patients with CNBD requiring specific immunohistochemistry). The cost of each terminal ileum histopathological assessment was then calculated by adding the fixed costs (a), (b) and (c) to the cost (d) multiplied by its variable frequency within the study population.

\section{Results}

A total of 546 patients with CNBD met the inclusion criteria and were consecutively enrolled between 2014 and 2017 at participating centers.

The ileal intubation rate was $97.6 \%$. Based on the exclusion criteria, 492 patients (209 men; mean age: $53 \pm 18$ years) were ultimately included in the statistical analysis (Figure 1). No endoscopic incidents or adverse events related to ileo-colonoscopy with biopsy occurred.

By combining the results achieved during the endoscopic and histopathological work-up, $79 \%$ of our study population had a fully negative diagnostic work-up, while $21 \%$ received a final diagnosis consistent with the history of CNBD (Supplementary Figure 1). The most prevalent entity was microscopic colitis, with 43 new cases detected (8.7\%), 28 collagenous and 15 lymphocytic colitis. Among them, the endoscopic inspection with HD plus virtual chromoendoscopy showed the presence of moderate (erosions, cat scratches) to mild (hyperemia, edema) colorectal inflammatory lesions in seven out of 43 cases $(16 \%)$. In the remaining $36 / 43$ patients, histopathology based on multiple random colon and rectum biopsies led to the definite diagnosis of microscopic colitis following a fully negative ileo-colonoscopy with HD plus virtual chromoendoscopy.

\section{Retrograde ileoscopy findings and accuracy}

Retrograde ileoscopy with HD and virtual chromoendoscopy revealed the features of lymphoid nodular hyperplasia in 34 patients (7\%). Histopathological analysis based on terminal ileum biopsy samples confirmed the endoscopic appearance of lymphoid nodular hyperplasia in all the cases (sensitivity/specificity 100\%). 


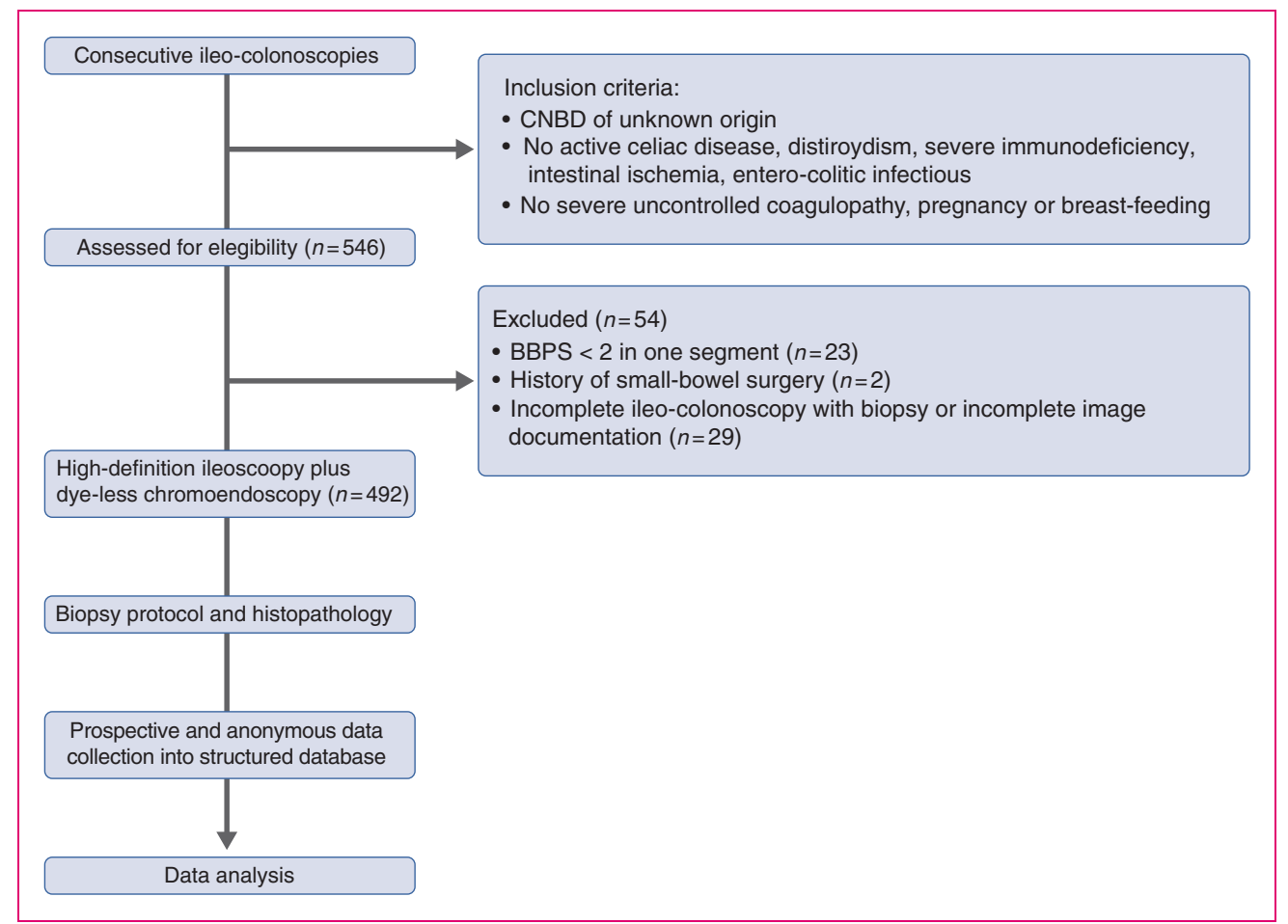

Figure 1. Study flowchart.

Apart from lymphoid nodular hyperplasia, retrograde ileoscopy identified inflammatory lesions in 22 patients $(4 \%)$ (Figure 2$) ; 15$ of whom $(3 \%)$ had a negative endoscopic inspection throughout their colon and rectum (i.e. isolated ileitis; Supplementary Figure 1).

Histopathology confirmed the presence of relevant inflammatory lesions in the terminal ileum samples of 14 patients with endoscopic ileitis and in one patient affected by severe Crohn disease located in the right colon showing severe cellular infiltrate beyond a normal-appearing terminal ileum (Table 1, Supplementary Figure 2). Based on the histopathological gold standard, the accuracy of retrograde ileoscopy with HD plus virtual chromoendoscopy imaging was $98.2 \%$. The corresponding performance measures of retrograde ileoscopy are shown in Table 2. Notably, the histopathological assessment based on multiple random biopsies of the terminal ileum had no clinical impact on the CNBD patients with a fully negative HD plus virtual chromoendoscopy ileo-colonoscopy inspection (i.e. sensitivity/specificity 100\%).

The use of digital (i-SCAN or Flexible SpectralImaging Color Enhancement (FICE)) and optical (NBI or SPIES) chromoendoscopy was equally distributed in our study cohorts $(46.1 \%$ and $53.9 \%$, respectively). However, the rate of patients with confirmed terminal ileitis was much greater in the digital chromoendoscopy group (12/15). Both optical and digital chromoendoscopy HD ileoscopy showed excellent accuracy levels
$(99.2 \%, \quad 95 \% \quad \mathrm{CI}=97.3 \%-99.9 \% ; \quad 96.9 \%, \quad 95 \%$ $\mathrm{CI}=93.8 \%-98.8 \%$, respectively) and a perfect negative predictive value $(100 \%, 95 \% \mathrm{CI}=98 \%-100 \%$, for both) using histopathology as the gold standard. The best sensitivity level was found using digital chromoendoscopy techniques (sensitivity: $100 \%, 95 \% \mathrm{CI}=74 \%-$ 100\%; specificity: $97 \%, 95 \% \mathrm{CI}=93 \%-99 \%$ ), while the best specificity was with optical chromoendoscopy (sensitivity: 67\%, 95\% CI 90\%-99\%; specificity: $100 \%, 95 \% \mathrm{CI}=99 \%-100 \%)$. Whether this figure reflects different features of optical and digital chromoendoscopy rather than center- or operator-driven factors cannot be definitively ascertained according to the observational design of this study and to the smaller prevalence of ileitis in the optical chromoendoscopy groups.

\section{Costs related to the terminal ileum histopathological assessment}

The estimated costs for standard slide preparation (including specimen embedding, sectioning, hematoxylin and eosin staining and pathology review) were $€ 15.25$ and $\$ 21.55$, respectively, at the Italian and US high-volume referral centers. Specific situations requiring additional sectioning of terminal ileum specimens for additional staining with immunohistochemistry and additional pathology review occurred every 10 CNBD patients (Table 3). All in all, the Italian and US costs 


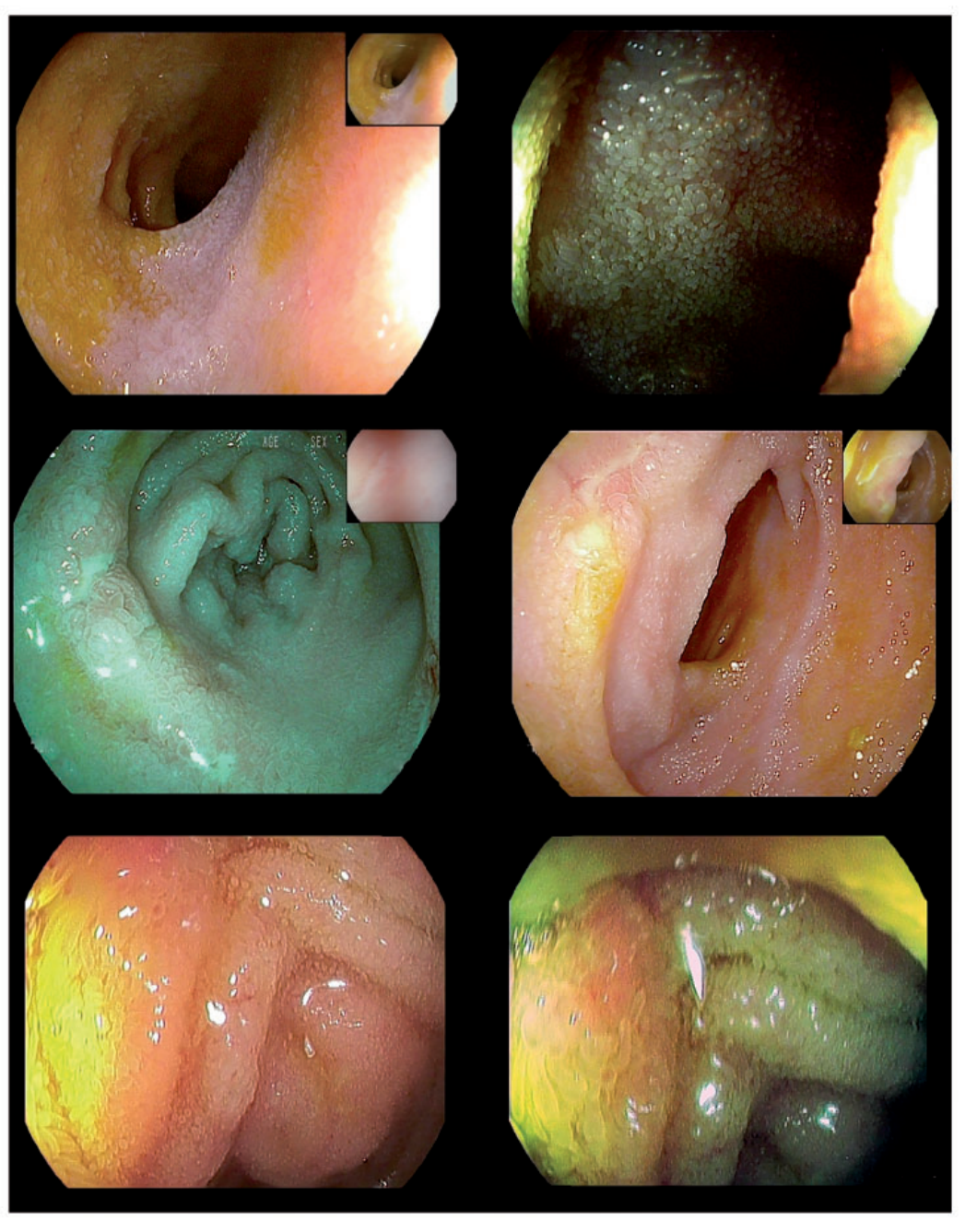

Figure 2. ( $a$ and b) High-definition plus i-SCAN mode 1 and i-SCAN mode 3 imaging of a normal-appearing terminal ileum as confirmed by histopathology on multiple random biopsy samples. (c and d) High-definition plus i-SCAN mode 1 and i-SCAN mode 2 imaging of small superficial ulcers diagnosed as Crohn disease ileitis by histopathology on targeted biopsies. (e and f) High-definition plus i-SCAN mode 1 and i-SCAN mode 2 imaging of mild hyperemia and edema of the terminal ileum in nonsteroidal anti-inflammatory drug-induced ileitis according to histopathology on targeted biopsies and clinical history.

Table 1. Detection of ileitis by means of ileo-colonoscopy with high-definition plus virtual chromoendoscopy and histopathological assessment.

\begin{tabular}{lccc}
\hline & Histopathology + & Histopathology - & Total \\
\hline lleoscopy + & 14 & 8 & 22 \\
Ileoscopy - & 1 & 469 & 470 \\
Total & 15 & 477 & 492 \\
\hline
\end{tabular}

directly related to the additional histopathological assessment of the terminal ileum in each patient performing colonoscopy with multiple colorectal biopsies were $€ 18.20$ and $\$ 26.50$, respectively.
Table 2. Statistical measures of the performance of retrograde ileoscopy with high-definition plus virtual chromoendoscopy using histopathology as the gold standard.

\begin{tabular}{lc}
\hline Test & Value \\
\hline Sensitivity & $0.933(0.660-0.996)$ \\
Specificity & $0.983(0.966-0.992)$ \\
Positive predictive value & $0.636(0.408-0.820)$ \\
Negative predictive value & $0.998(0.986-1)$ \\
Positive likelihood ratio & $55.6(27.6-112.1)$ \\
Negative likelihood ratio & $0.068(0.010-0.450)$
\end{tabular}


Table 3. Cost analysis of additional histopathological assessment of the terminal ileum for every Italian and United States (US) patient undergoing colonoscopy with multiple colorectal biopsies for chronic nonbloody diarrhea.

\begin{tabular}{|c|c|c|c|}
\hline Additional procedure & Workflow & $\begin{array}{l}\text { Italian cost } \\
\text { in Euros }(€)\end{array}$ & $\begin{array}{l}\text { US cost in } \\
\text { dollars (\$) }\end{array}$ \\
\hline Endoscopic sampling & $\begin{array}{l}\text { Terminal ileum biopsies (two to four bites) placed in a separate } \\
\text { disposable container preloaded with buffered formalin }\end{array}$ & 0.25 & 0.25 \\
\hline Standard slide preparation & Specimen embedding, sectioning, hematoxylin and eosin staining & 10.00 & 12.00 \\
\hline Pathology review & Reading, analysis and report drafting & 5.00 & 9.30 \\
\hline Special situations ${ }^{a}$ & $\begin{array}{l}\text { Specimens sectioned for two additional immunohistochemistry } \\
\text { staining and pathology review }\end{array}$ & 30.00 & 50.00 \\
\hline Total cost & & 18.25 & 26.55 \\
\hline
\end{tabular}

${ }^{a}$ Special situations occurred every 10 patients on average.

\section{Discussion}

CNBD of unknown origin is one of the major indications for gastrointestinal endoscopy and accounts for approximately $4 \%-7 \%$ of all the colonoscopies performed in Western countries. ${ }^{19-21}$ Multiple endoscopic biopsies and histopathological assessment represent an essential step to ascertain the diagnostic definition of macroscopic lesions and exclude the underlying presence of microscopic colitis. ${ }^{9,22}$ The diagnostic yield of colonoscopy with biopsies in CNBD patients ranges from $7 \%$ to $32 \%$, with microscopic colitis and IBD being most common. ${ }^{1-5,8-10,16-18}$ The present multicenter study, conducted in a real-life setting, identified a relevant number of patients suffering from microscopic colitis $(9 \%)$, IBD $(6 \%)$, adenocarcinoma $(1 \%)$ and other inflammatory conditions corresponding to the clinical history of CNBD (Supplementary Figure 1).

Our study has confirmed that retrograde ileoscopy can be safely accomplished in up to $98 \%$ of colonoscopies, resulting in the detection of visible ileitis in $4 \%$ of patients suffering from CNBD. Interestingly, a definite diagnosis of inflammatory illness with isolated involvement of the terminal ileum was achieved in $3 \%$ of all the CNBD patients, who would have otherwise been dismissed with the wrong putative diagnosis of irritable bowel syndrome (IBS) (Supplementary Figure 1). However, retrograde ileoscopy is not yet accepted among the standard quality indicators for colonoscopy, ${ }^{23-25}$ and it is often regarded as a matter for referral to IBD endoscopy units. Several retrospective studies in real-life clinical settings have clearly shown that retrograde ileoscopy is performed in only $45 \%$ to $87 \%$ 3,8,26-28 of patients with chronic diarrhea. By contrast, prospective studies aimed at performing ileal intubation in all cases have shown higher success rates $(90 \%-95.5 \%),{ }^{3,17}$ thereby suggesting ample room for improvement in everyday clinical practice.

The value of random biopsies routinely performed on the terminal ileum of patients with CNBD and negative ileo-colonoscopy dates back to the era of low-resolution endoscopic imaging, when subtle mucosal lesions could have been overlooked. Within this setting, the histopathological assessment of normalappearing mucosa of the terminal ileum was found positive for relevant small-bowel pathologies, including isolated Crohn disease and lymphoma, in up to $4 \%$ of patients with chronic diarrhea of unknown origin. $^{5,7-9,29}$ In 2010, the Standards-of-Practice Committee of the ASGE stated that the yield of biopsy of an endoscopically normal-appearing terminal ileum is likely to be low and highlighted the lack of evidence to determine whether this practice should be routinely performed on patients with chronic unexplained diarrhea. ${ }^{1}$ More recently, anecdotal cases of microscopic ileitis have also been described in diverted-enteric-segment and collagenous colitis. ${ }^{30}$ However, the clinical relevance of microscopic ileitis is still unknown in non-IBD patients.

Hence, we assessed for the first time the accuracy of retrograde ileoscopy with HD and virtual chromoendoscopy using histopathology as the gold standard. Our results have clearly showed that ileoscopy with advanced imaging can predict the presence of microscopic ileitis in CNBD patients with excellent accuracy. Consistently, routine endoscopic biopsy of the terminal ileum had no diagnostic yield in CNBD patients with fully negative ileo-colonoscopy using HD imaging and virtual chromoendoscopy, and it should be avoided except for specific indications, thereby resulting in $\$ 26.50$ saved per patient (Table 3).

By contrast, histopathology based on multiple random biopsies of the colon and rectum is crucial for the diagnosis of microscopic colitis regardless of the presence of visible inflammatory lesions. Consistent with the literature, ${ }^{19,22,31}$ most patients diagnosed with microscopic colitis $(84 \%)$ in the present study had a fully negative ileo-colonoscopy, even despite the use of HD and virtual chromoendoscopic imaging. 
The application of rigorous inclusion and exclusion criteria for patient enrollment together with the prospective nature of this multicenter cohort study represent an optimal methodological background to support our results. However, some points regarding this study should be addressed. First, two dedicated subspecialtytrained gastrointestinal pathologists were systematically involved in all histopathological analyses at each tertiary referral center but no external revision by blinded pathologists was performed for research purposes. Second, no case of adenocarcinoma or lymphoma of the terminal ileum was detected, resulting in no opportunity to assess the performance of HD plus virtual chromoendoscopy in oncologic disorders. However, these conditions are quite uncommon in patients with $\mathrm{CNBD},{ }^{32}$ and are usually associated with clear endoscopic abnormalities given their erosive nature. Third, as far as the study protocol was concerned, our results could not assess the additional impact of virtual chromoendoscopy beyond HD white-light imaging. In other settings, the routine use of advanced endoscopic imaging was found to increase the detection and the operators' confidence in characterizing and delineating subtle inflammatory lesions, such as isolated erosions, hyperemia and/or edema. ${ }^{10-13}$ Finally, no long-term follow-up was designed to evaluate whether those patients with a negative endoscopic and histological work-up achieved symptom remission or eventually had any diagnosis of other disorders consistent with CNBD. In these cases, we hypothesized the functional origin of the diarrhea with the putative diagnosis of IBS or colonic diverticular disease.

In conclusion, this large, prospective, multicenter study has clearly shown that retrograde ileoscopy with HD imaging and virtual chromoendoscopy can predict the presence of ileitis in patients with CNBD with excellent accuracy. Following negative ileo-colonoscopy inspection, routine histopathological assessment of the terminal ileum has substantial costs and no clinical impact on the diagnostic work-up of CNBD, except for specific indications such as immune-compromised patients, suspicion of intestinal tuberculosis, human immunodeficiency virus infection or graft-vs-host disease. By contrast, histopathology based on multiple biopsies is crucial for the diagnostic definition of microscopic colitis and of any visible inflammatory lesion detected during ileo-colonoscopy for CNBD.

\section{Acknowledgments}

The authors are indebted to Marco Maggioni (MD, Pathology Unit, Fondazione IRCCS Ca' Granda Ospedale Maggiore Policlinico, Milan, Italy) and to Carlo De Salvo (PhD, Department of Pathology, Case Western Reserve University School of Medicine, Cleveland, $\mathrm{OH}$,
USA) for their contribution to the cost analyses included in the present study.

Author contributions include the following: E.B.: acquisition of data, analysis and interpretation of data, drafting and critical revision of the manuscript. B.B., R.D.I., F.C., L.P., E.R., L.S., C.V. and M.V.: acquisition of data and critical revision of the manuscript for important intellectual content. G.B.: analysis and interpretation of data and critical revision of the manuscript for important intellectual content. H.N.: study concept and design and critical revision of the manuscript for important intellectual content. G.E.T.: study concept and design, acquisition of data, analysis and interpretation of data, drafting of the manuscript, critical revision of the manuscript and study supervision.

\section{Declaration of conflicting interests}

None declared.

\section{Funding}

G.E.T. has received a grant from the Italian Society of Gastroenterology (Premio SIGE 2014) supporting his involvement in this research project.

\section{Ethics approval}

All patient records (inclusion/exclusion criteria, endoscopic and histopathological findings) were anonymized and prospectively collected in a structured database. Each center received formal approval from the local ethics committee before study initiation. This study was carried out in accordance with the Declaration of Helsinki adopted in 1964 and incorporating all later amendments.

\section{Informed consent}

All patients gave their informed written informed consent prior to ileo-colonoscopy for the present trial.

\section{ORCID iD}

Edoardo Borsotti (D) https://orcid.org/0000-0003-2318-7384

\section{References}

1. Shen B, Khan K, Ikenberry S, et al. The role of endoscopy in the management of patients with diarrhea. Gastrointest Endosc 2010; 71: 887-892.

2. Geboes K. The strategy for biopsies of the terminal ileum should be evidence based. Am J Gastroenterol 2007; 102: 1090-1092.

3. Morini S, Lorenzetti R, Stella F, et al. Retrograde ileoscopy in chronic nonbloody diarrhea: A prospective, casecontrol study. Am J Gastroenterol 2003; 98: 1512-1515.

4. Lengeling RW, Mitros FA, Brennan JA, et al. Ulcerative ileitis encountered at ileo-colonoscopy: Likely role of nonsteroidal agents. Clin Gastroenterol Hepatol 2003; 1: 160-169.

5. Geboes K, Ectors N, D'Haens G, et al. Is ileoscopy with biopsy worthwhile in patients presenting with symptoms of inflammatory bowel disease? Am J Gastroenterol 1998; 93: 201-206. 
6. Tontini GE, Vecchi M, Pastorelli L, et al. Differential diagnosis in inflammatory bowel disease colitis: State of the art and future perspectives. World $J$ Gastroenterol 2015; 21: 21-46.

7. McHugh JB, Appelman HD and McKenna BJ. The diagnostic value of endoscopic terminal ileum biopsies. Am J Gastroenterol 2007; 102: 1084-1089.

8. Yusoff IF, Ormonde DG and Hoffman NE. Routine colonic mucosal biopsy and ileoscopy increases diagnostic yield in patients undergoing colonoscopy for diarrhea. $J$ Gastroenterol Hepatol 2002; 17: 276-280.

9. Shah RJ, Fenoglio-Preiser C, Bleau BL, et al. Usefulness of colonoscopy with biopsy in the evaluation of patients with chronic diarrhea. Am J Gastroenterol 2001; 96: 1091-1095.

10. Tontini GE, Rath T and Neumann H. Advanced gastrointestinal endoscopic imaging for inflammatory bowel diseases. World J Gastroenterol 2016; 22: 1246-1259.

11. Kiesslich R and Neurath MF. Advanced endoscopy imaging in inflammatory bowel diseases. Gastrointest Endosc 2017; 85: 496-508.

12. Waldner MJ, Rath T, Schürmann S, et al. Imaging of mucosal inflammation: Current technological developments, clinical implications, and future perspectives. Front Immunol 2017; 8: 1256.

13. Subramanian V, Ramappa V, Telakis E, et al. Comparison of high definition with standard white light endoscopy for detection of dysplastic lesions during surveillance colonoscopy in patients with colonic inflammatory bowel disease. Inflamm Bowel Dis 2013; 19: 350-355.

14. Cotton PB, Eisen GL, Aabakken L, et al. A lexicon for endoscopic adverse events: Report of an ASGE workshop. Gastrointest Endosc 2010; 71: 446-454.

15. Langner C, Aust D, Ensari A, et al. Histology of microscopic colitis-review with a practical approach for pathologists. Histopathology 2015; 66: 613-626.

16. Magro F, Langner C, Driessen A, et al. European consensus on the histopathology of inflammatory bowel disease. J Crohns Colitis 2013; 7: 827-851.

17. Zammit SC, Cachia M, Sapiano K, et al. Eosinophilic gastrointestinal disorder: Is it what it seems to be? Ann Gastroenterol 2018; 31: 475-479.

18. Villanacci V, Casella $G$ and Bassotti G. The spectrum of drug-related colitides: Important entities, though frequently overlooked. Dig Liver Dis 2011; 43: 523-528.

19. Tontini GE, Pastorelli L, Spina L, et al. Microscopic colitis and colorectal neoplastic lesion rate in chronic nonbloody diarrhea: A prospective, multicenter study. Inflamm Bowel Dis 2014; 20: 882-891.

20. Meral M, Bengi G, Kayahan H, et al. Is ileocecal valve intubation essential for routine colonoscopic examination? Eur J Gastroenterol Hepatol 2018; 30: 432-437.

21. Patel Y, Pettigrew NM, Grahame GR, et al. The diagnostic yield of lower endoscopy plus biopsy in nonbloody diarrhea. Gastrointest Endosc 1997; 46: 338-343.

22. Pardi DS. Diagnosis and management of microscopic colitis. Am J Gastroenterol 2017; 112: 78-85.

23. Rees CJ, Thomas Gibson S, Rutter MG, et al. UK key performance indicators and quality assurance standards for colonoscopy. Gut 2016; 65: 1923-1929.

24. Rizk MK, Sawhney MS, Cohen J, et al. Quality indicators common to all GI endoscopic procedures. Am J Gastroenterol 2015; 81: 3-16.

25. Kaminski M, Thomas-Gibson S, Bugajski M, et al. Performance measures for lower gastrointestinal endoscopy: A European Society of Gastrointestinal Endoscopy (ESGE) Quality Improvement Initiative. Endoscopy 2017; 5: 309-334.

26. Makkar R, Lopez R and Shen B. Clinical utility of retrograde terminal ileum intubation in the evaluation of chronic non-bloody diarrhea. J Dig Dis 2013; 14: 536-542.

27. Jeong $\mathrm{SH}$, Lee $\mathrm{KJ}$, Kim $\mathrm{YB}$, et al. Diagnostic value of terminal ileum intubation during colonoscopy. $J$ Gastroenterol Hepatol 2008; 23: 51-55.

28. Koksal AR, Boga S, Alkim H, et al. How does a biopsy of endoscopically normal terminal ileum contribute to the diagnosis? Which patients should undergo biopsy? Libyan $J$ Med 2014; 9: 23441.

29. Sayilir A, Kurt M, Kekilli M, et al. Diagnostic value of terminal ileum biopsy in chronic diarrhea with normal endoscopic appearance. J Dig Dis 2011; 12: 188-192.

30. Zuo C, Fu Z, Lee EC, et al. Microscopic ileitis in diverted and nondiverted enteric segments: An underrecognized condition with a multifactorial etiology. Hum Pathol 2018; 77: 80-87.

31. Marlicz W, SkoniecznaŻdecka K, Yung DE, et al. Endoscopic findings and colonic perforation in microscopic colitis: A systematic review. Dig Liver Dis 2017; 49: 1073-1085.

32. Pan SY and Morrison H. Epidemiology of cancer of the small intestine. World J Gastrointest Oncol 2011; 3: 33-42. 